\title{
Fotodesbridamento de Válvulas Aórticas Calcificadas com Laser de $\mathrm{CO}_{2}$
}

\author{
Walter José GOMES*, Saul GOLDENBERG*, Ênio BUFFOLO*
}

RBCCV 44205-27

\begin{abstract}
GOMES, W. J.; GOLDENBERG, S.; BUFFOLO, E - Fotodesbridamento de válvulas aórticas calcificadas com laser de $\mathrm{CO}_{2}$. Rev. Bras. Circ. Cardiovasc., 2(2): 93-97, 1987.

RESUMO: Os autores estudam 6 válvulas aórticas calcificadas retiradas de pacientes operados de troca valvar aórtica, submetidos a fotodesbridamento com raios laser de $\mathrm{CO}_{2}$. Os estudos radiográficos, fotográficos, histopatológicos e pesagens realizadas revelam ter sido o fotodesbridamento extenso, resultado em válvulas restauradas morfológica e funcionalmente. Mostram as vantagens do método quando comparado às substituições da válva por prótese e prevêem aplicação clínica para este método.

DESCRITORES: laser, plastia valvular; fotodesbridamento, válvulas cardiacas; válvulas cardiacas, cirurgia.
\end{abstract}

\section{INTRODUÇĀO}

Poucas vezes na história da Medicina, uma inovação na terapêutica das doenças despertou tanto interesse e curiosidade como o desenvolvimento do laser. A aplicabilidade do laser no campo médico e, particularmente, cardiovascular, tem crescido de maneira progressiva e segura, superando problemas de ordem tecnológica e operacional.

LASER é o nome formado pelo conjunto das iniciais de: "Light Amplification by Stimulated Emission of Radiation" 6 , e seu princípio foi delineado por EINSTEIN, em $1916^{11}$.

Em 1960, MAIMAN ${ }^{14}$ relatou a construção do primeiro aparelho de laser, usando cristal de rubi. Imediatamente após o desenvolvimento do laser, iniciaram-se pesquisas sobre sua aplicação nos campos da Biologia e Medicina. Trabalhos pioneiros com o uso do laser, no campo da cirurgia cardiovascular, foram realizados no Instituto de Cirurgia Memorial A. V. Vishinevsky, de Moscou, por ARAPOV et alii ${ }^{1}$, em 1974 . Foram realizadas comissurotomias a laser de válvulas pulmonares estenóticas, obtendo-se conformaçōes semilunares das cúspides semelhantes às normais.
No Brasil, em 1980, MACRUZ et alii ${ }^{13}$ empregaram - laser de argônio para destruir placas de ateromas de retalhos de aorta, retiradas de pacientes idosos.

ISNER et alii ${ }^{9}{ }^{10}$, em 1984 e 1985, empregaram, pela primeira vez, o laser de $\mathrm{CO}_{2}$ no desbridamento de válvulas aórticas calcificadas, obtidas de cadáveres, ou removidas cirurgicamente. Trabalhando com válvulas aórticas em que a estenose, por calcificação das cúspides, era o substrato anatomopatológico, conseguiram remoçōes adequadas dos depósitos de cálcio, produzindo folhetos com mobilidade próxima ao normal.

O objetivo do presente trabalho é verificar a factibitidade do fotodesbridamento de válvulas aórticas com extensos depósitos de cálcio com o uso do raio laser de $\mathrm{CO}_{2}$.

\section{MATERIAL E MÉTODO}

$\mathrm{Na}$ realização deste trabalho, foram utilizadas seis válvulas aórticas calcificadas retiradas de pacientes submetidos a cirurgia de troca aórtica.

\footnotetext{
Trabalho realizado na Disciplina de Cirurgia de Tórax da Escola Paulista de Medicina. São Paulo, SP, Brasil. Apresentado ao 14: Congresso Nacional de Cirurgia Cardiaca. Salvador, BA, 27 e 28 de março, 1987.

- Da Escola Paulista de Medicina.

Endereço para separatas: Walter José Gomes. Rua Jaci, 54, Apto. 43. Saúde, 04140. São Paulo, SP. Brasil.
} 
GOMES. W. J.: GOLDENBERG. S.: BUFFOLO. E - Fotodesbridamento de valvulas aorticas calcificadas com laser de CO . Rev Bras. Circ. Cardiovasc. 2(2):93-97. 1987

Destes pacientes, 3 eram do sexo masculino e 3 do sexo feminino, com idades variando entre 41 e 63 anos (média de idade de 53,3 anos). Quatro pacientes apresentavam estenose aórtica isolada e 2, dupla disfunção aórtica, tendo sido operados no Hospital São Paulo da Escola Paulista de Medicina e no Hospital do Coração da Associação do Sanatório Sírio, entre outubro de 1985 e janeiro de 1986. As disfunçōes valvulares aórticas foram todas confirmadas por estudo cineangiográfico e pressórico.

As válvulas retiradas cirurgicamente em monobloco foram preservadas em soro fisiológico a $4^{\circ} \mathrm{C}$ de temperatura.

Na etapa de pré-desbridamento, as válvulas aórticas calcificadas foram submetidas a pesagem em balança eletrônica, com aproximação de 3 casas decimais; documentação radiográfica foi levada a efeito na Disciplina de Radiologia do Departamento de Diagnóstico por Imagem da Escola Paulista de Medicina e documentação fotográfica, realizada no laboratório de fotografia da Disciplina de Tórax da Escola Paulista de Medicina.

Na etapa de pós desbridamento, o material foi novamente submetido a pesagem, documentação radiográfica e fotográfica e estudo histopatológico.

Dos espécimes retirados, 4 valvas aórticas eram tricúspides e 2 eram bicúspides por afecção congênita.

Para a realização do fotodesbridamento foi utilizado um equipamento de raio laser de $\mathrm{CO}_{2}$, o Sharplan 733 . pertencente ao Laboratório da Unidade Experimental de Raio Laser do Instituto de Ciências Biomédicas da Universidade de São Paulo.

O aparelho Sharplan 733 é um laser de $\mathrm{CO}_{2}$ de construçāo simples, que permite um ajuste focal mínimo, tem grande precisão e apresenta uma uniformidade de distribuição de energia sobre a área de trabalho. Possui um módulo de sustentação com painel de controle e um braço articulado onde o feixe luminoso produzido caminha por meio de espelhos especialmente dispostos no seu interior.

O braço articulado permite grande mobilidade ao cirurgīāo, tendo, em sua extremidade, a "caneta cirúrgica", para onde o feixe luminoso refletido é dirigido. A ativação do aparelho é feita por um disparador eletrônico em forma de pedal.

Foram utilizados no fotodesbridamento das válvulas aórticas calcificadas potências compreendidas entre 5 e $8 \mathrm{~W}$, sempre no modo continuo.

A técnica empregada para o desbridamento envolveu a incisão térmica do envelope de colágeno que revestia os depósitos de cálcio das válvulas aórticas, com movimentos seguidos e contínuos da "caneta cirúrgica" sobre estes depósitos. Uma vez estabelecido um plano tecidual entre o colágeno envolvendo o cálcio e o tecido fibroso nảo calcificado da válvula, eram utilizadas pinças atraumáticas delicadas para a retirada dos blocos calcificados. Geralmente, era necessário o uso intermitente do laser para completar a dissecçâo e excisâo das calcificaçōes, com o aparecimento, em seguida, da superfície regular do folheto.

\section{RESULTADOS}

A Tabela 1 mostra os dados obtidos com a pesagem pré e pós desbridamento das válvulas aórticas calcificadas com o uso do raio laser de $\mathrm{CO}_{2}$.

TABELA 1

\begin{tabular}{cccc}
\hline Caso No & Peso Pré $(\mathrm{mg})$ & Peso Pós $(\mathrm{mg})$ & $\mathrm{P}^{*}(\mathrm{mg})$ \\
\hline 1 & 4,541 & 1,351 & 3,190 \\
\hline 2 & 4,060 & 0,965 & 3,095 \\
\hline 3 & 3,493 & 0,930 & 2,563 \\
\hline 4 & 3,011 & 0,419 & 2,592 \\
\hline 5 & 2,593 & 0,683 & 1,910 \\
\hline 6 & 1,554 & 0,339 & 1,215 \\
\hline
\end{tabular}

'peso pré - peso pós desbridamento.

Podemos notar, a partir dos dados da Tabela 1, que houve uma reduçâo importante nos pesos pós desbridamento, em relação aos pré desbridamentos, denotando a extensa retirada dos blocos de cálcio.

$\mathrm{Na}$ Tabela 2, onservamos as potências utilizadas na aplicação do laser com o aparelho Sharplan 733 .

TABELA 2

\begin{tabular}{cc}
\hline Caso N: & Potência Utilizada \\
\hline 1 & $5 \mathrm{~W}$ \\
\hline 2 & 5 e $8 \mathrm{~W}$ \\
\hline 3 & 5 e $8 \mathrm{~W}$ \\
\hline 4 & $5 \mathrm{~W}$ \\
\hline 5 & $5 \mathrm{~W}$ \\
\hline 6 & $5 \mathrm{~W}$ \\
\hline
\end{tabular}


GOMES. W. J.: GOLDENBERG. S.: BUFFOLO, E - Fotodesbridamento de válvulas aorticas calcificadas com laser de $\mathrm{CO}_{2}$. Rev. Bras. Circ. Cardiovasc. 2(2):93-97, 1987

As potências utilizadas no fotodesbridamento foram baixas, compreendidas entre 5 e $8 \mathrm{~W}$, sempre no modo continuo.

A seguir, apresentamos as documentaçōes fotográficas, radiográficas e histopatológicas de 1 caso estudado (Figuras 1 a 5 ).

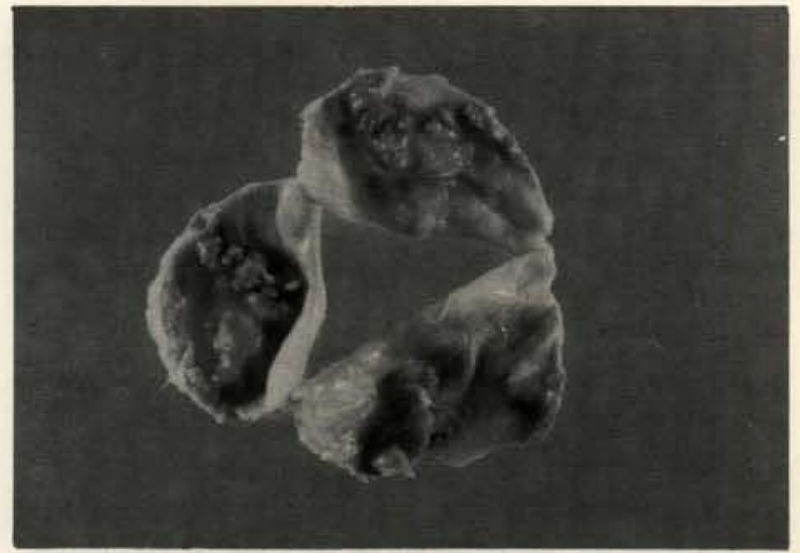

Fig. 1 - Aspecto pré desbridamento, com a válvula exibindo grandes blocos de cálcio deformando a estrutura dos folhetos e com vegetaçōes grosseiras

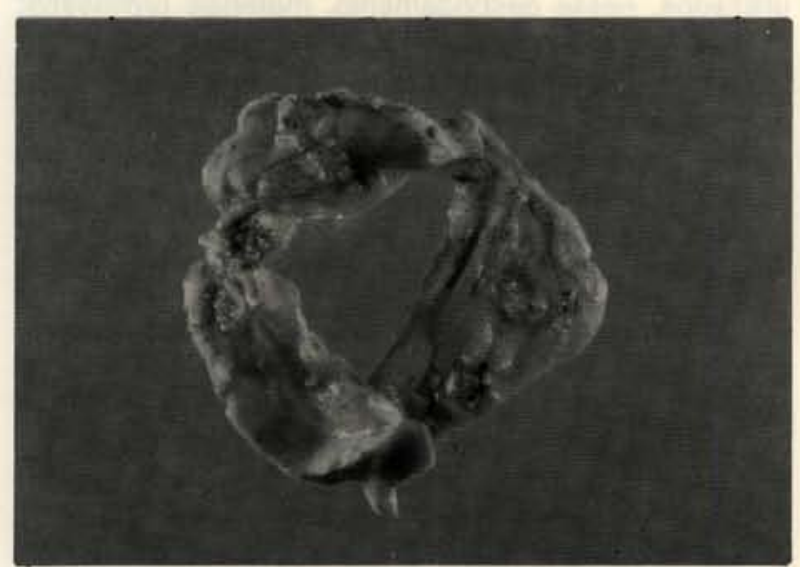

Fig. 2 - Aspecto pós desbridamento mostra a válvula já livre dos blocos de cálcio

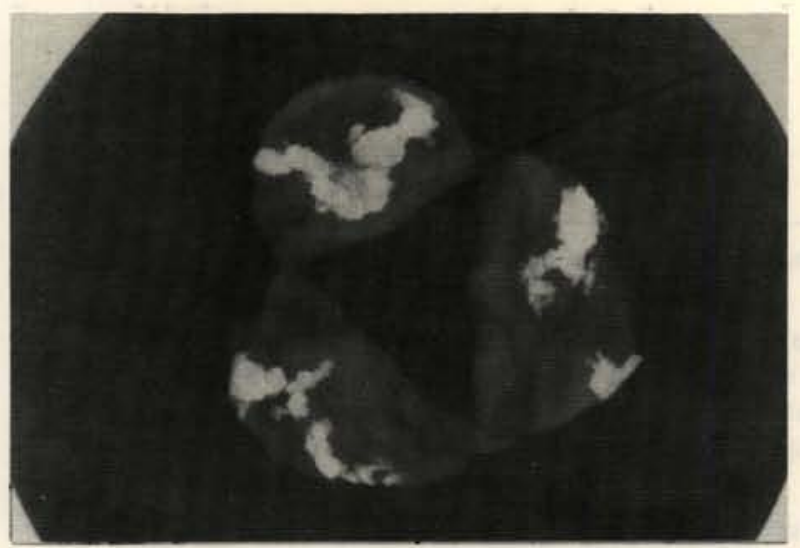

Fig. 3-Radiografia pré desbridamento documentando os blocos de cálcio presentes na estrutura valvular.

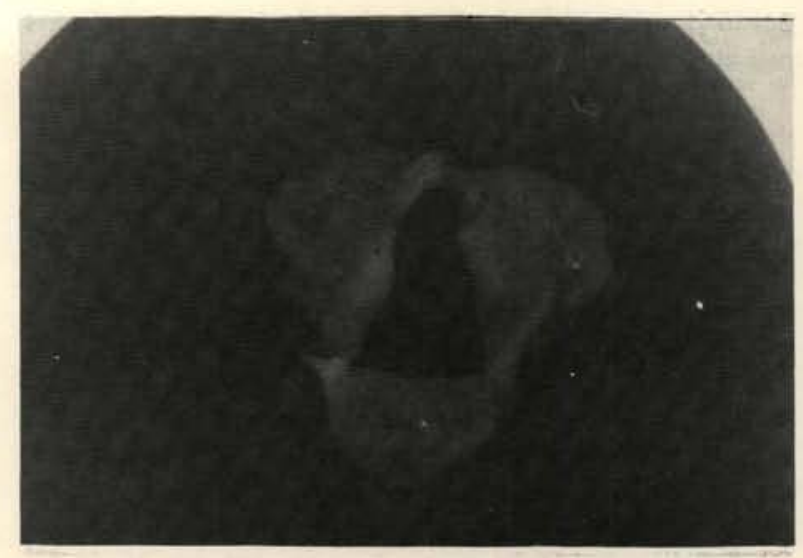

Fig. 4-Radiografia pós desbridamento exibindo a válvula isenta dos blocos de cálcio.

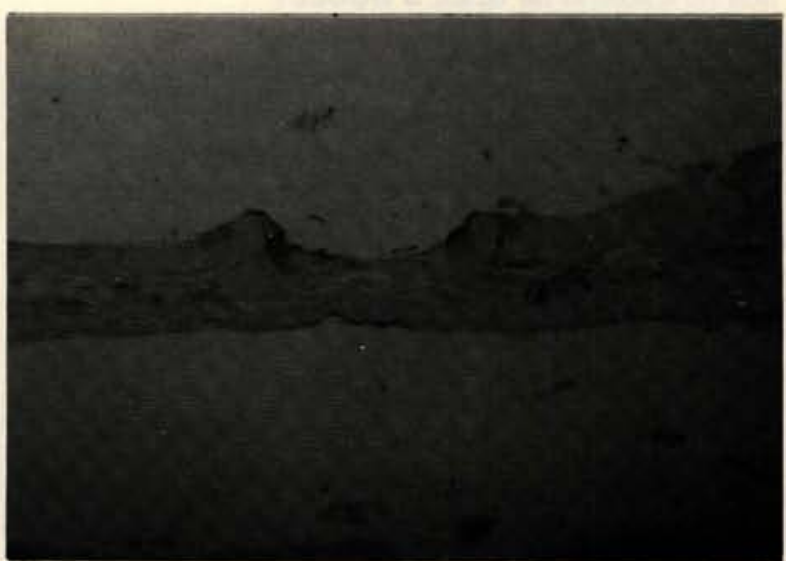

Fig. 5 - Estudo histopatológico da válvula desbridada, mostrando espessamento das cúspides as custas de proliferação de tecido fibrilar denso com hialinizaçāo. Detalhe da "cratera" resultante da ação da luz do laser.

\section{DISCUSSÃO}

Depósitos de cálcio sobre os folhetos de válvulas aórticas constituem a base patológica única para a estenose valvular, em grande número de pacientes. As válvulas são estenóticas simplesmente porque os folhetos estão imobilizados por extensos depósitos de cálcio, impedindo sua livre excursão. Por outro lado, depósitos de cálcio também são freqüentes na evolução das doenças valvulares de etiologia reumática. Em muitos casos, se o cálcio puder ser retirado sem lesāo valvular irreversível, a estenose poderá ser reduzida a graus hemodinamicamente insignificantes.

A aplicaçāo do fotodesbridamento de válvulas aórticas calcificadas beneficia-se de um achado anatomopatológico fortuito: o cálcio nas válvulas aórticas doentes está, basicamente, depositado na face aórtica (em oposição à ventricular) dos folhetos. Isto implica em que, do 
GOMES. W. J.: GOLDENBERG, S.: BUFFOLO. E - Fotodesbridamento de válvulas aorticas calcificadas com laser de $\mathrm{CO}_{2}$. Rev. Bras. Circ. Cardiovasc., 2(2):93-97, 1987

ponto de vista técnico, é possivel desbridar os folhetos por abordagem trans-aórtica.

Por outro lado, os depósitos de cálcio estão mais freqüentemente confinados à porção fibrosa superficial do folheto, sendo incomum que esses depósitos se extendam para a porção esponjosa profunda ${ }^{5}$.

seyundo ROBERTS et alii ${ }^{15}{ }^{16}$, sāo comuns, em pacientes acima de 65 anos de idade, o aparecimento de sopro sistólico aórtico e depósitos de cálcio na válvula aórtica, podendo resultar em extensas calcificaçōes valvulares e estenose aórtica severa, sem fusão comissural, somente pela imobilização funcional dos folhetos. Não se conhece, até o momento, a real incidência deste tipo de patologia, já que não há achados para justificar uma etiologia reumática. Achados semelhantes estão incluídos no trabalho de SELL \& SCULLY ${ }^{17}$.

BAILEY et alii ${ }^{2,3}$ provaram ser tecnicamente possivel a remoção intra-operatória a céu aberto dos depósitos de cálcio dos folhetos de válvulas aórticas e demonstraram as vantagens da restauraçāo da flexibilidade valvular no tratamento cirúrgico de estenose aórtica calcificada, apesar de utilizarem, para este fim, simplesmente manipulação de materiais grosseiros. Trabalhos subseqüentes, de KIRKLIN \& MANKIN ${ }^{12}$, em 1960, e HUFNAGEL \& CONRAD ${ }^{8}$, em 1962, corroboraram os resultados obtidos por Bailey.

A escolna do raio laser de $\mathrm{CO}_{2}$ contribuiu para a obtenção dos resultados apresentados, visto que o mesmo provoca verdadeiras explosōes celulares, facilitando a separação dos tecidos e retirada dos blocos de cálcio.

As vantagens da utilização intra-operatória de fotodesbridamento das válvulas aórticas calcificadas, em relação aos métodos convencionais atualmente utilizados de troca valvar por prótese, incluem a manutenção, pelo paciente, de sua própria válvula, evitando as complicaçōes inerentes a essas próteses, a necessidade e o risco associado da utilização, por longo tempo, da medicação anticoagulante e a evidência da experiência inicial indicar a necessidade relativa de pouco tempo de circulação extracorpórea (aproximadamente 20 minutos), para a realização do fotodesbridamento. Como vantagem adicional, mencionamos a maior área valvar efetiva de fluxo, quando comparada à cirurgia de implante de prótese aórtica.

Pelo presente trabalho, o uso do laser de $\mathrm{CO}_{2}$ mostrou ser possivel a retirada dos depósitos de cálcio das válvulas aórticas, sendo um método adequado sempre quando seja possivel uma boa exposição da válvula e ela possa ser livremente manipulada, como mostram os estudos radiológicos obtidos após o desbridamento.

Embora não tenha sido possivel realizar estudos hemodinâmicos, na fase de pós desbridamento, que comprovem ser a fibrose e a calcificação residuais insignificantes, do ponto de vista hemodinâmico, existem evidências que justificam tal inferência: 1 ) a realização de desbridamento, nas décadas de 50 e 60 , conforme relatos de Bailey, Kirklin e Hufnagel, mostra documentada melhora clínica e hemodinâmica, apesar de terem sido utilizados, nesse desbridamento, materiais grosseiros 2) aparente boa mobilidade e flexibilidade à inspecção manual dos folhetos; 3 ) pacientes com moderada fibrose ou calcificação dos folhetos da válvula aórtica, que nunca exibem sintomatologia; e também 4) a prótese valvular produz uma obstrução ao fluxo, como mostram os estudos pós-operatórios ${ }^{4}{ }^{7}$.

Finalmente, os estudos fotográficos, radiológicos, anatomopatológicos e pesagens realizadas mostraram ser a fibrose e a calcificação residuais mínimas, tendo havido um extenso e muito satisfatório fotodesbridamento das válvulas aórticas calcificadas. Estes achados são encorajadores, em termos de possibilidade de uma longa duração desses folhetos, já que houve restauração às suas características morfológicas e funcionais próprias, vislumbrando-se, assim, uma imediata aplicaçâo clínica.

\section{RBCCV 44205-27}

GOMES, W. J.; GOLDENBERG, S.; BUFFOLO, E. - Photodebridement of calcified aortic valve, with $\mathrm{CO}_{2}$. laser. Rev. Bras. Cir. Cardiovasc., 2(2): 93-97, 1987

ABSTRACT: The authors present 6 cases of calcified aortic valves, removed from patients during aortic valve replacement, which underwent photodebridement with $\mathrm{CO}_{2}$ laser. Radiographic, photografic weight and histopathological studies have shown extensive debridement, and the valves showing improved morphology and function. The advantages of the procedure made concerning valve replacement and clinical application is foreseen for the method. surgery.

DESCRIPTORS: laser, cardiac valve replacement; photodebridement, cardiac valves; cardiac valves, 
GOMES, W. J.; GOLDENBERG. S.; BUFFOLO, E - Fotodesbridamento de válvulas aórticas calcificadas com laser de $\mathrm{CO}_{2}$. Rev. Bras. Circ. Cardiovasc., 2(2):93-97, 1987

\section{REFERÊNCIAS BIBLIOGRÁFICAS}

1 ARAPOV, A. D.: VISHNEVSKY, A. A.; ABDULLAEV, F. Z.; KORCHA, GIN, V. A.; MIRTSKHULAVA, K. A.; SARGIN, M. E. - Pervyi opyt primeneniia lazernogo lucha v kardiokhirurgii. Eksp. Khir. Anesteziol, 4: 10-12, 1974.

2 BAILEY, C. P.; BOLTON, H. E.; NICHOLS, H. T.; JAMIESON, W. L.; LITWAK, R. S. - The surgical treatment of aortic stenosis. J. Thoracic Surg., 31 (4): 375-437, 1956.

3 BAILEY, C. P. \& LIKOFF, W. - Surgical management of aortic stenosis: evaluation of techniques and results. Arch. Int. Med., 99 (6): 859-887, 1957.

4 CHAITMAN, B. R. - Hemodinamic evaluation of the Carpentier-Edwards porcine xenograft. Circulation, 60: (5): $1170-1178,1979$

5 FINEGAN, R. E.; GIANELLY, R. E.; HARRISON, D. C. - Aortic stenosis in the elderly: relevance of age to diagnosis and treatment. N. Eng. J. Med. 281 (23): 1261-1264, 1969.

6 GOLDENBERG, A.; GOLDENBERG, S.; CHACON, J. P. - O laser de $\mathrm{CO}_{2}$ na diérese e na hemostasia. An. Paul. Med. Cir., 111 (4): 39-44, 1984.

7 HENRY, W. L.; BONOW, R. O.; BORER, J. S.; KENT, K. M.; WARE, J. H.; REDWOOD, D. R.; ITSCOITZ, S. B.; MCINTOSH, C. L.; MORROW, A. G.; EPSTEIN, S. E. - Evaluation of aortic valve replacement in patients with valvular aortic stenosis. Circulation, 61 (4): 814-825, 1980.

8 HUFNAGEL, C. A. \& CONRAD, P. W. - Calcific aortic stenosis. N. Eng. J. Med., 266 (2): 72, 1962.

9 ISNER, J. M.; MICHLEWITZ, H.; CLARKE, R. H.; DONALDSON, R. F.; KONSTAM, M. A.; SALEM, D. N. - Laser assisted debridement of aortic valve calcium. Am. Heart J., 109 (3) : 448-452, 1985.

10 ISNER, J. M.; MICHLEWITZ, H.; CLARKE, R. H.; DONALDSON, R. F.; SALEM, D. N. - Laser photoablation of aortic valve calcium: evaluation of argon versus carbon-dioxide lasers using excised surgical and post mortem specimens. J. Am. Coll. Cardiol., 3 (4): 558-562, 1984.

11 KASTLER, A. - Birth of the laser and maser. Nature, 316 (25): 307-309, 1985.

12 KIRKLIN, J. W. \& MANKIN, H. T. - Open operation in the treatment of calcific aortic stenosis. Circulation, 21 (4): 578-586, 1960.

13 MACRUZ, R.; MARTINS, J. R. M.; TUPINAMBÁ, A. S.; LOPES, E. A.; VARGAS, H.; PENA, A. F.; CARVALHO, V. B.; ARMELIN, E.; DÉCOURT, L. V. - Possibilidades terapêuticas do raio laser em ateromas. Arq. Bras. Cardiol., 34 (1): 9-12, 1980.

14 MAIMAN, T. H. - Stimulated optical radiation in ruby. Nature, 187 (27): 493-494, 1960.
15 ROBERTS, W. C. - The structure of the aortic valve in clinically isolated aortic stenosis: an autopsy study of 162 patients over 15 years of age. Circulation, 42 (1): 91-97, 1970.

16 ROBERTS, W. C.; PERLOFF, J. K.; CONSTANTINO, T. - Severe valvular aortic stenosis in patients over 65 years of age. Am. J. Cardiol., 27 (1): 497-506, 1971.

17 SELL, S. \& SCULLY, R. E. - Aging changes in the aortic and mitral valves: histologic and histochemical studies. with observations on the patogenesis of calcific aortic stenosis and calcification of the mitral annulus. $\mathrm{Am}$. J. Pathol., 46 (3): 345-354, 1965.

\section{Discussão}

\section{DR. PAULO M. PÊGO-FERNANDES \\ São Paulo, SP}

Gostaria, inicialmente, de agradecer à Comissão Organizadora a indicaçāo do nosso nome para comentar este excelente trabalho e de agradecer aos colegas da Divisão Experimental do Instituto do Coração, pelos subsídios fornecidos a respeito do tema. A experiência do uso do laser, no Instituto do Coraçāo, é baseada no laser argônio e, previamente, foram publicados vários trabalhos com a aplicação desse método, em vários tecidos cardiacos, sendo realizados dois estudos sobre valvas cardiacas; a valvotomia pulmonar e o encurtamento de cordas tendineas da valva mitral. Em todas as aplicações com laser, um dos problemas foi a dificuldade de padronização da carga e o tempo de aplicação. Gostaria de perguntar, inicialmente, ao Dr. Walter: como foi feita a padronização da energia usada neste estudo? O Dr. Lee e colaboradores, em 1984, produziram insuficiência aórtica aguda com laser argônio com potência de $5 \mathrm{~W}$ e exposição de 5 segundos. Os cirurgiōes que realizam desbridamento de válvulas aórticas calcificadas conhecem o risco de perfuração de suas válvulas. Gostaria, finalmente, de perguntar: qual o risco de perfuraçāo acidental de válvulas por energia demasiada e se existe algum parâmetro que possamos considerar para sustar a aplicação do laser antes que isto ocorra?

\section{DR. GOMES}

(Encerrando)

Dr. Lee e colaboradores realizaram, experimentalmente, em cães, perfuração da válvula aórtica, utilizando laser de argônio dirigido por catéter de fibra ótica. Nós realizamos nosso trabalho "a céu aberto", usando laser de $\mathrm{CO}_{2}$, que, devido ao seu elevado comprimento de onda, não se transmite em meios líqüidos, minimizando o risco de perfuração. Este risco existe, mas não ocorreu em nenhum material de nosso estudo. Talvez isto se devesse à incidência do feixe da luz laser sobre o espesso encasing de colágeno envolvendo o bloco calcificado: A padronização da energia foi feita, experimentalmente, em estudos anteriores. 